\title{
Bone Metastases in Neuroendocrine Tumors: Molecular Pathogenesis and Implications in Clinical Practice
}

\author{
Mauro Cives ${ }^{a}$ Eleonora Pellè ${ }^{a}$ Maria Rinzivillo ${ }^{b}$ Daniela Prosperi ${ }^{c}$ \\ Marco Tucci $^{\mathrm{a}}$ Franco Silvestris $^{\mathrm{a}}$ Francesco Panzuto $^{\mathrm{b}}$ \\ ${ }^{a}$ Department of Biomedical Sciences and Human Oncology, University of Bari Aldo Moro, Bari, Italy; ${ }^{b}$ Digestive \\ Disease Unit, Sant' Andrea University Hospital, ENETS Center of Excellence, Rome, Italy; ' Nuclear Medicine Unit, \\ Sant' Andrea University Hospital, ENETS Center of Excellence, Rome, Italy
}

\section{Keywords \\ Carcinoid tumors · Skeleton-related events . \\ Bisphosphonates · Denosumab · CXCR4}

\begin{abstract}
Skeletal colonization is often regarded as a rare event in patients with neuroendocrine tumors (NETs) although both national registries and retrospective series report an incidence of bone metastases as high as $20 \%$ in subjects with advanced disease. While the biological mechanisms leading to bone metastatic colonization in NETs have been poorly investigated so far, key steps of osteotropic mechanisms, including the epithelial-to-mesenchymal transition, preparation of the premetastatic niche, migration of circulating tumor cells towards the bone marrow as well as the resulting alterations of the skeletal metabolism, are likely to operate also during the development of NET bone metastases. The skeleton involvement by NETs has a detrimental impact on both quality of life and patients' prognosis, leading to pain in the majority of symptomatic subjects. While it is currently unclear whether or not the earlier recognition of bone involvement by PET/CT imaging techniques employing ${ }^{68} \mathrm{Ga}$-DOTA-conjugated peptides might improve outcomes through the ex-
\end{abstract}

karger@karger.com

(c) 2020 S. Karger AG, Basel

www.karger.com/nen

Karger" ploitation of timely treatments, the management of bonecolonizing NETs is today based only on clinical experience from other osteotropic tumors. Here, we summarize the fundamental molecular mechanisms driving bone colonization and revisit both established and novel treatments for patients with bone metastatic NETs.

(c) 2020 S. Karger AG, Basel

\section{Introduction}

Neuroendocrine tumors (NETs) are heterogeneous neoplasms arising in secretory cells of the diffuse neuroendocrine system. They predominate in the gastroenteropancreatic (GEP) tract and the bronchopulmonary (BP) tree and are characterized by a relatively indolent rate of growth and the ability to release a variety of peptide hormones and vasoactive substances [1]. The incidence of NETs has increased markedly over the last three decades, probably as a result of trends in imaging and improvement in diagnosis, and the projected prevalence of NETs in the US population in 2014 was 171,321 [2]. The clinical aggressiveness of NETs widely varies in relation to their primary site as well as the tumor grading. According to 
the 2019 World Health Organization (WHO) classification, well-differentiated NETs are subdivided as either G1, G2 or G3 tumors, whereas poorly differentiated carcinomas are always considered G3 and have a dismal prognosis [3].

NETs are frequently diagnosed at stage IV, with liver and peritoneum being the most common sites of metastasis [2,4-7]. Skeletal colonization is often regarded as a rare event in patients with NETs, and retrospective series reported the incidence of bone metastases as high as $20 \%$ in patients with advanced disease [7-10]. However, the clinical advent of PET/CT imaging techniques employing ${ }^{68} \mathrm{Ga}$-DOTA-conjugated peptides has substantially increased the frequency of NET bone metastasis detection, thus allowing their earlier recognition [7]. As result, a progressive rise in the incidence of bone metastatic disease is expected in patients with NETs in the next few years. Notably, multiple studies have depicted the bone colonization as a negative prognostic factor in NETs while skeleton-related events (SREs) including pathological fractures, spinal cord compression, need for orthopedic surgery or radiotherapy to bone and/or hypercalcemia, are reported to occur in approximately one fifth of patients with bone-colonizing NETs [7-14]. Despite their clinical relevance, the management of NET bone metastases is currently guided by limited evidence, and treatment patterns vary substantially among different institutions.

Here, we summarize the fundamental molecular mechanisms driving bone colonization in NETs and critically discuss the diagnostic and therapeutic opportunities currently available for NET patients with bone metastatic disease.

\section{Epidemiology of Bone Metastases in NETs}

The incidence of bone metastases in patients with NETs has been investigated only by retrospective series so far. In an analysis of the Swedish Cancer Registry [4], skeletal involvement has been identified in the $16 \%$ of 1,842 patients with metastatic NETs diagnosed between 1987 and 2012, in the presence of a substantial male preponderance. In a study including 14,685 patients with gastrointestinal NET from the Surveillance, Epidemiology and End Results database, bone metastases were recorded in $6 \%$ of them with a significantly higher incidence in males [15]. A similar frequency of skeletal involvement was described in a study of the Spanish National Cancer Registry, as well as in a French multi- center study prospectively enrolling 668 patients with GEP-NET $[6,16]$. Institutional series from tertiary academic centers in the USA reported an incidence of bone metastatic disease in approximately $12 \%$ of patients $[8,9]$, consistent with a recent single-institution German study assessing 677 patients diagnosed with NET between 2000 and 2015 [7]. Notably, a 72\% relative rise in the frequency of bone metastasis detection has been reported in this study, where the incidence of skeletal involvement among patients with the metastatic disease increased from 19 to $33 \%$ in two distinct periods in which both Octreoscan and ${ }^{68} \mathrm{Ga}$-DOTATOC PET/CT imaging techniques were exclusively used [7]. Furthermore, in a recent study of 162 metastatic lung carcinoids, the bone involvement was observed in $42 \%$ of cases [17], while a frequency of $38 \%$ was reported in a single-institution series enrolling 108 patients with metastatic BP-NETs [14]. Overall, the incidence of NET bone metastases appears to be higher in lung carcinoids as compared with GEP-NETs, probably as result of their different venous drainage. Consistently, rectal NETs appear to have the highest osteotropic potential in the context of GEP-NETs [4]. Epidemiological inconsistencies among different retrospective studies may be related to referral patterns, while underreporting cannot be excluded in national registries. Evidence from a small autopsy series [18], reporting a bone metastasis incidence of $42 \%$ in 36 subjects with carcinoid tumors, supports the idea that the frequency of skeletal involvement might be clinically underestimated in patients with NETs.

\section{Bone Metastases in NETs: Molecular Pathogenesis}

Bone metastatic colonization is a complex multistep process including four distinct phases (Fig. 1). In the first phase, cancer cells in the primary tumor site acquire the ability to invade surrounding tissue and spread. In the second phase, primary tumor cells influence the bone marrow stromal cells via soluble factors, thus preparing the so-called premetastatic niche. In the third phase, cancer cells migrate as circulating tumor cells (CTCs) towards skeletal premetastatic niches under the pressure of a specific transcriptional rewiring or as result of cytokine gradients. In the fourth phase, tumor cells colonize the skeleton and enter a state of dormancy, or aberrantly modulate bone resorption or deposition leading to the development of lytic or sclerotic metastasis, respectively [19].

Similarly to other cancers, NET cells may acquire invasive potential through the epithelial-to-mesenchymal 


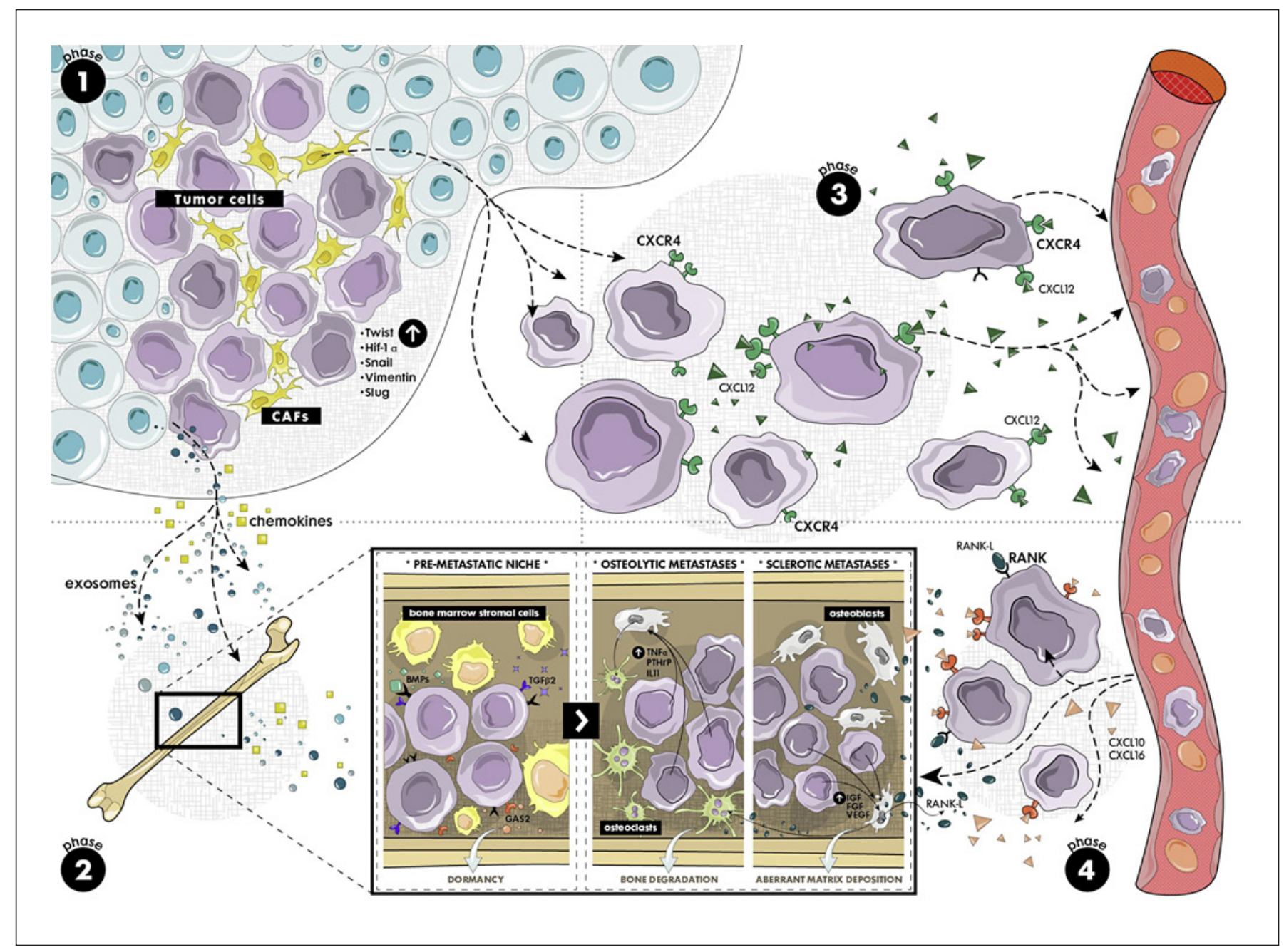

Fig. 1. The process of bone metastatic colonization proceeds through multiple steps. Under the pressure of a supportive microenvironment, tumor cells in the primary site undergo epithelialto-mesenchymal transition as result of a complex transcriptional rewiring coordinated by transcription factors including snail, slug or twist, among others (phase 1). Concomitantly, malignant cells prepare the so-called premetastatic niche through a variety of soluble factors, thus priming the nursing ability of bone marrow stromal cells to support the tumor (phase 2). After intravasation into the tumor vasculature, circulating tumor cells migrate towards bone marrow niches as result of specific interactions between marrow-derived cytokines such as CXCL12 or RANKL and their cognate receptors expressed on the surface of cancer cells (phase 3).
Once settled in the metastatic niches, cancer cells may either enter a state of dormancy, as indolent micrometastasis, or proliferate and aberrantly modulate bone metabolism, leading to the development of lytic or sclerotic metastases (phase 4). CAFs, cancer-associated fibroblasts; CXCR4, C-X-C chemokine receptor type 4; CXCL12, CXCL10, CXCL16, C-X-C motif chemokine ligands 12, 10,16 ; RANK, receptor activator of nuclear factor- $\kappa \mathrm{B}$; RANKL, receptor activator of nuclear factor- $\kappa \mathrm{B}$ ligand; BMPs, bone morphogenetic proteins; TGF- $\beta_{2}$, transforming growth factor- $\beta_{2}$; GAS2, growth arrest-specific 2; TNF- $\alpha$, tumor necrosis factor- $\alpha$; PTHrP, parathyroid hormone-related protein; IL-11, interleukin-11; IGF, insulin-like growth factor; FGF, fibroblast growth factor; VEGF, vascular endothelial growth factor. transition (EMT), a transdifferentiation program conferring mesenchymal plasticity to epithelial cells [20]. Multiple studies have demonstrated that the EMT drives the progression of both well- and poorly differentiated NETs, and that EMT-undergone tumors have a dismal prognosis, as demonstrated by the association between poor out- comes and the overexpression of EMT-related factors including snail, slug, twist and vimentin in tumor cells [2127]. In this context, bone-colonizing NETs have been shown to harbor specific EMT-related features. In an immunohistochemistry study of 44 samples of GEP- or BP-NETs, osteotropic tumors appeared to overexpress 
C-X-C chemokine receptor type 4 (CXCR4) in the presence of low levels of TGF- $\beta_{1}$ as compared with localized neoplasms [11]. In this regard, in vitro evidence suggests that agonist stimulation of CXCR 4 by its ligand CXCL12 is able per se to induce EMT in NET cell lines, enhancing their migratory and invasive capabilities towards bone tissue [28]. Intriguingly, the pharmacological suppression of the CXCL12/CXCR4 axis by ulocuplumab, a CXCR4 targeting monoclonal antibody, has been preliminarily shown to inhibit EMT in pancreatic NET (panNET) cells, and to downmodulate their metastatic potential both in vitro and in vivo [29].

Intratumor hypoxia has been described as a major driver of EMT in NETs. Gene expression profiling of the GOT1 carcinoid cell line revealed a marked upregulation of EMT-related genes under hypoxic conditions [30], and the activation of EMT has been demonstrated in panNETs arising in the context of Von Hippel-Lindau disease, a syndrome caused by germline mutations of $V H L$, a critical regulator of the oxygen sensor HIF-1 $\alpha$ [31]. While it is well known that different components of the tumor microenvironment play a crucial role in enhancing cancer invasiveness and metastatic spreading, little is understood in this regard in NETs. Preliminary evidence from our group indicates that cancer-associated fibroblasts stimulate both EMT and in vitro migration of NET cells, while tumor-infiltrating platelets, a key regulator of EMT, have been recently demonstrated to predict poor outcomes and early relapse in patients with resected panNETs [32].

Systemic signals produced by the primary tumor can modulate the microenvironment of distant organs, creating a pre-metastatic niche prior to the arrival of CTCs. Extensive research (reviewed in Peinado et al. [33]) has demonstrated that both inflammatory chemokines, proangiogenic cytokines, extracellular matrix-remodeling enzymes as well as extracellular vesicles such as exosomes can induce in distant organs the formation of a microenvironment conducive to the survival and outgrowth of disseminated tumor cells, dictating selective tumor organotropism [34]. Although it is likely that the mechanisms driving the osteotropism of NETs are similar to those operating in other cancers, no studies have specifically addressed the question of whether and how neuroendocrine primary tumors are able to prepare the bone premetastatic niche. In this context, the peripheral trafficking of bone marrow-derived stem cells, a key component of the premetastatic niche, appears to be more limited in gastric NETs as compared with gastric adenocarcinoma, consistent with their lower metastatic potential [35].
During the process of metastatic colonization, tumor cells are progressively shed into the systemic circulation as CTCs. Based on available evidence, relatively low numbers of CTCs can be isolated in patients with either GEPor BP-NETs, and the presence of CTCs has been shown to independently predict increased tumor burden and poor survival outcomes [36-38]. In a study of 254 patients with GEP-NETs, the presence of CTCs was significantly associated with metastatic skeletal colonization, but not with lung, peritoneal or lymph node metastases [39]. Given that cancer cells are able to remain as "niche-engaged" dormant cells in the bone marrow for years before switching to a proliferative phenotype and eventually causing overt metastases, the identification of CTCs has been proposed as a useful tool for tailoring bone-oriented surveillance programs in patients with NETs.

Several factors are suspected to influence the bone tropism of CTCs in NETs. First, circulation patterns dictate the first capillary bed that CTCs will encounter, and therefore it is not surprising that lung carcinoids display a higher incidence of skeletal metastases as compared with GEP-NETs, which are drained by the mesenteric stream. Second, specific chemokines including CXCL12, CXCL10, CXCL16 and receptor activator of nuclear factor- $\kappa B$ ligand (RANKL) may attract CTCs towards the osteogenic niche. In this context, in an analysis of $40 \mathrm{pa}-$ tients with metastatic NETs, the expression of CXCR4 on the surface of CTCs was apparently associated with an osteotropic behavior ( $p=0.18$ ) [39]. Third, definite patterns of transcriptional remodeling have been described to drive the homing of CTCs towards the skeleton [40]. In this regard, preliminary evidence suggests that acquisition of the EMT gene signature primes the skeletal colonization in NETs [29].

Once they have reached the bone metastatic niche, disseminated tumor cells may either enter a quiescent state or evolve in overt metastases [40]. Little is known about how NET cells enter the dormant state, what signals sustain it, what niches these dormant cells inhabit and, more importantly, what molecular cues trigger the resumption of aggressive tumor growth eventually leading to overt metastasis formation. Bone morphogenetic proteins, TGF- $\beta_{2}$ and growth arrest-specific- 6 protein have been reported to enforce the quiescence of disseminated tumor cells in a variety of malignancies [40], but their specific role in NETs is currently unknown. When the bone microenvironment becomes suitable for cancer cell proliferation, lytic or sclerotic bone metastases may arise. Osteolytic metastases result from an altered balance between bone-resorbing osteoclasts and bone-generating osteo- 
blasts. Cancer cell-derived parathyroid hormone-related protein, interleukin-11 and tumor necrosis factor- $\alpha$ stimulate osteoblasts to release RANKL, which promotes osteoclast maturation and activation, leading to bone degradation. In turn, growth factors physiologically stored in the bone matrix are released during this process, reigniting cancer cell proliferation and skeletal devastation in a vicious cycle. In sclerotic metastases, cancer cells produce osteoblast-stimulating factors including fibroblast growth factor, vascular endothelial growth factor and insulin-like growth factor, thus driving aberrant bone matrix deposition [41]. In patients with NETs, sclerotic bone metastases appear to be more prevalent as compared with lytic ones [11], but the exact molecular mechanisms leading to their development are presently unknown. Similarly to other bone-devastating malignancies, an imbalance of the RANKL/osteoprotegerin serum levels has been described in patients with NETs with bone metastatic disease as compared with those without skeletal colonization, and the measurement of these factors has been proposed as a predictor of skeletal colonization in clinical practice [42].

\section{Bone Metastases in NETs: Clinical Features, Diagnostic Workup and Prognostic Impact}

The diagnosis of bone lesions in patients with NETs is steadily increasing, mainly due to the improved performance of radiological and nuclear medicine diagnostic techniques [43, 44]. Most frequently, NET bone metastases appear osteosclerotic and involve with higher frequency the axial rather than the appendicular skeleton [7, 8,11 . While bone involvement is detected incidentally in up to $40 \%$ of patients with NETs, clinical complications of NET bone metastases have been observed in approximately half of the cases, with no correlation detected between SRE occurrence and gender, radiological appearance of bone lesions, tumor primary site and WHO grade $[9,11]$. Reportedly, pain is the most frequent symptom in patients with NET bone metastases and can severely impact the quality of life in a population of long-surviving patients. In this regard, an analysis of 85 patients with NETs metastatic to the skeleton showed that bone pain was reported by 28 and $14 \%$ of subjects at the initial diagnosis and during the follow-up, respectively [7]. On the other hand, fractures have been described as rare events, occurring in nearly $10-20 \%$ of cases, and even lower rates of spinal compression (5\%) and hypercalcemia (1-4\%) have been reported $[7,45]$. Although no clear factor able to predict the development of NET bone metastases has been identified, a correlation with deteriorated performance status has been observed [10]. Of note, a similar prevalence of bone lesions and their complications has been noted in patients with pancreatic versus gastrointestinal NETs $[9,10]$.

Both conventional and functional imaging techniques are commonly used for the diagnosis of bone metastases in NET patients. Although in the presence of contrasting evidence $[7,8,11,13,46]$, MRI appears to have a higher sensitivity in identifying NET bone lesions as compared with CT, and particularly whole-body MRI scans with diffusionweighted imaging sequences seem to have high diagnostic accuracy in this context (Fig. 2). The ability to detect skeletal metastases has dramatically increased since the introduction of ${ }^{68} \mathrm{Ga}$-PET/CT, which clearly outperforms both bone scintigraphy and somatostatin receptor scintigraphy (OctreoScan). Overall, the diagnostic accuracy of ${ }^{68} \mathrm{Ga}$ $\mathrm{PET} / \mathrm{CT}$ in identifying NET bone lesions is comprised between 80 and $100 \%$ according to recent studies [43, 47-49]. Notably, ${ }^{68} \mathrm{Ga}$-PET/CT imaging adds a significant diagnostic yield to conventional radiological procedures and may change NET management in a substantial proportion of patients. Compared to contrast-enhanced CT and MRI, ${ }^{68} \mathrm{Ga}$-PET/CT may provide a $20-25 \%$ yield in terms of diagnostic ability to detect bone metastases [43, 48, 50, 51]. False-positive results with ${ }^{68} \mathrm{Ga}-\mathrm{PET} / \mathrm{CT}$ may occur in patients with meningiomas, angiomas, lymphomas or inflammatory processes involving the skeleton, whereas false-negative findings may be associated with low or absent expression of somatostatin receptors by tumor cells.

The detection of bone metastases is associated with a worsening in patients' clinical outcome. Significantly lower progression-free survival (PFS) and overall survival rates have been reported in patients with skeletal involvement compared with those without bone lesions. In a large retrospective analysis from a part of the US National Comprehensive Cancer Network database including 691 patients, significantly lower survival rates were observed in low-grade NETs arising from both pancreas and small bowel [9]. Similar findings have been reported by other studies performed in ENETS Centers of Excellence across Europe, confirming the negative prognostic impact of bone metastases on patients' survival [7, 10, 45]. Nevertheless, in a study enrolling 312 consecutive patients with sporadic, grade $1 / 2$, stage IV, nonfunctioning panNETs, the presence of bone lesions predicted a shorter PFS, in the absence of any impact on overall survival [52]. Overall, median survival time from bone metastases identification ranges from 20 to 60 months [ $7,9,10,45,49]$, a figure that highlights the negative impact of this specific metastatic 

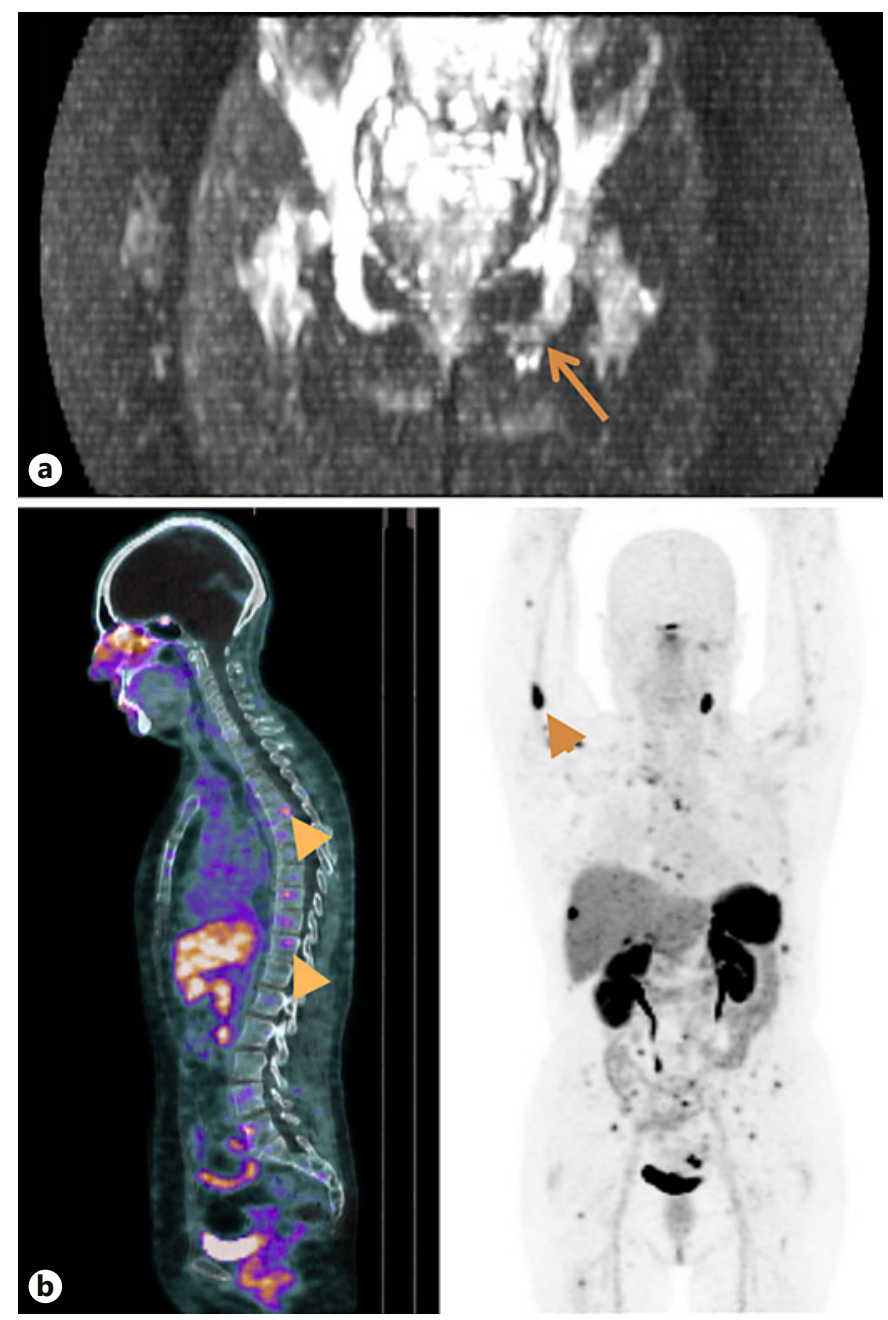

Fig. 2. The role of imaging techniques in detecting bone metastases in NET patients. a Multi-field-of-view whole-body MRI shows a skeletal metastasis in the right ischiopubic ramus of a patient with pancreatic NET (arrow). b A ${ }^{68} \mathrm{Ga}-\mathrm{PET} / \mathrm{CT}$ scan shows multiple axial and appendicular bone lesions in a patient with small bowel neuroendocrine tumor (arrowheads).

pattern compared with patients with stage IV disease without skeletal colonization, where median survival rates are usually comprised between 80 and 100 months [7, 9, 53]. Consistently, poorer survival outcomes have been reported for NET patients diagnosed with synchronous bone metastases as compared with those presenting metachronous skeletal colonization [11, 13]. Interestingly, bone metastases seem to have a major impact on the survival of patients with pancreatic NETs as compared with those with intestinal NETs [10], confirming that NETs from different sites should be considered separate entities in terms of tumor behavior and patient's clinical outcome.
Table 1. Key points for approaching neuroendocrine tumor (NET) patients with bone metastases

Bone metastases are not rare, occurring in up to $20 \%$ of stage IV NETs

They are asymptomatic in approximately $50 \%$ of patients Skeletal fractures and hypercalcemia are rare events

${ }^{68} \mathrm{Ga}-\mathrm{PET} / \mathrm{CT}$ is mandatory to correctly stage bone disease Bone metastases have a negative impact on patients' clinical outcomes

Bone-directed symptomatic therapies (surgery, radiotherapy, bisphosphonates and denosumab) may help preventing skeletal complications

Skeletal colonization predicts poor survival also in lung NETs, irrespective of the tumor grade or the synchronous/ metachronous presentation [14]. Key points to be attended in daily clinical practice when facing NET patients with bone metastases are outlined in Table 1 .

\section{Treatment of NET Bone Metastases}

Despite the availability of guidelines focusing on NET management published by international scientific societies, the therapeutic approach to bone metastases in the clinical practice is not well established yet. Two major goals need to be taken into account when facing NET patients with advanced disease and bone metastases: (i) systemic therapy of metastatic disease aimed at controlling tumor growth; (ii) bone-directed therapy aimed at reducing the risk of skeletal complications while improving the patient's quality of life. With regard to the first point, bone colonization has been described as a predictor of poor PFS in patients with advanced, well-differentiated GEP-NETs treated with first-line somatostatin analogs [54], as well as in patients with progressive NETs undergoing treatment with the mammalian target of rapamycin inhibitor everolimus [55]. On the other hand, promising results have been reported in studies investigating the efficacy of peptide receptor radionuclide therapy (PRRT) in NET patients with bone metastases. To date, PRRT is considered a reference therapy for well-differentiated, progressive, somatostatin receptor-expressing, advanced GEP-NETs, thanks to the impressive gain in terms of PFS obtained in comparison with high doses of octreotide long-acting release in the NETTER-1 trial [56]. In two single-institution, retrospective series from the University of Bonn, PRRT determined objective responses (complete/major/minor responses) in skeletal lesions in up to $50 \%$ of NET patients 


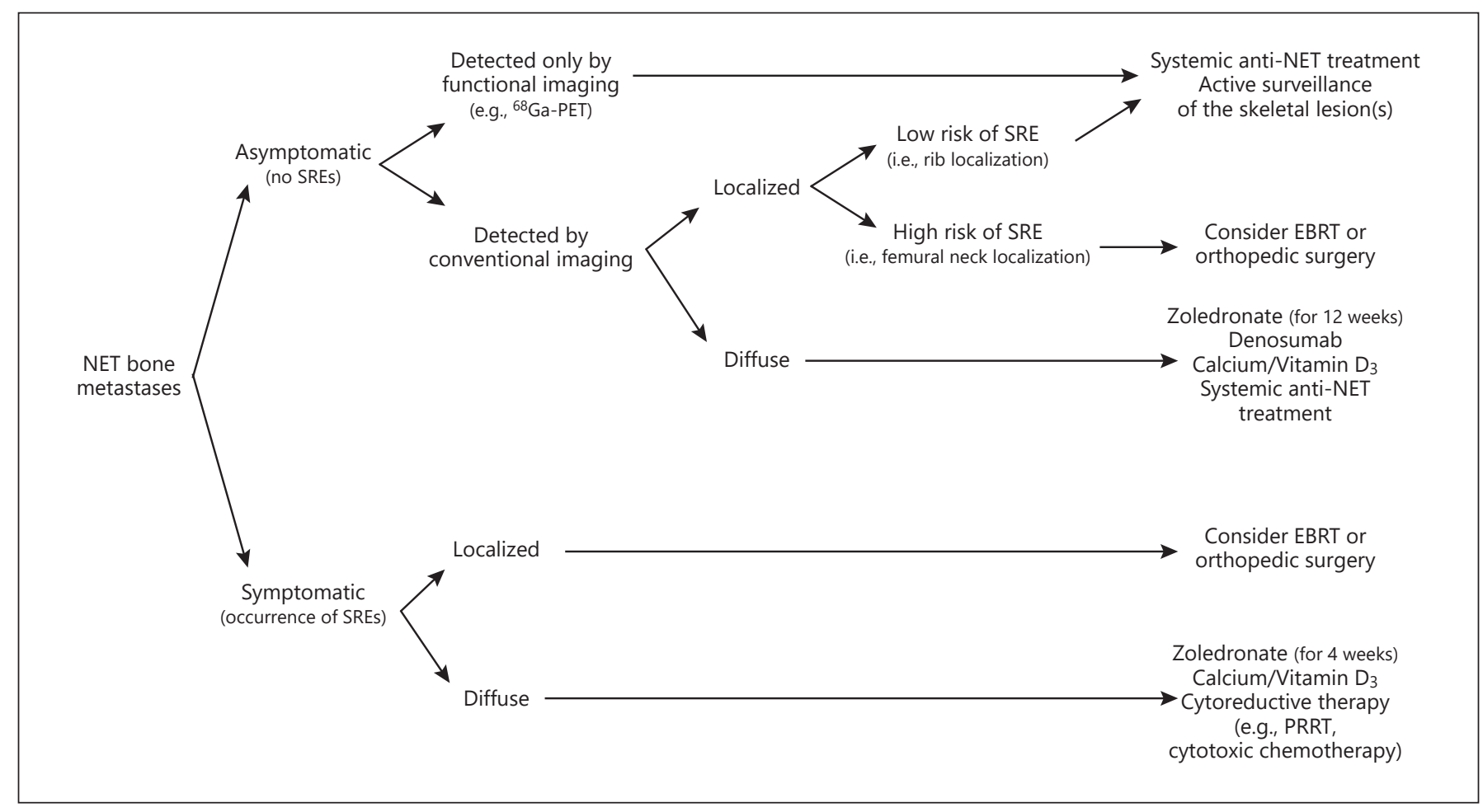

Fig. 3. Treatment tailoring in patients with neuroendocrine tumor (NET) bone metastases: a proposal for a therapeutic algorithm. SRE, skeleton-related event; EBRT, external beam radiotherapy; PRRT, peptide receptor radionuclide therapy.

with bone metastases [57,58], although in the presence of a higher risk of myelotoxicity [59]. Nevertheless, lower rates of objective responses to PRRT have been documented for NET bone metastases in other studies, and discrepancies in treatment response between bone and softtissue lesions have been described [60]. To the best of our knowledge, no studies have specifically investigated the impact of sunitinib or chemotherapy on NET bone metastases. Overall, an accurate assessment of bone disease in NET patients at diagnosis and throughout the course of the disease might be helpful in categorizing tumor clinical aggressiveness, thereby supporting physicians in the process of treatment personalization.

Bone-directed therapies include radiotherapy, orthopedic surgery, bisphosphonates (administered either orally or intravenously), and denosumab. Both radiotherapy and surgery are often used to prevent or treat SREs in patients with NET [11], and bone pain relief has been described in up to $90 \%$ of patients treated with radiotherapy [61]. In a study of 45 NET patients receiving palliative external beam radiotherapy, skeletal metastases represented the $75 \%$ of target lesions, and the median symptom-free survival was 4 months [62]. Main acute toxicities of external beam radiotherapy include erythema, loss of appetite, fatigue, nausea and vomiting, irrespective of the adoption of a single-dose or fractionated treatment schema [63]. Although their use is recommended by the ENETS guidelines [64], the administration of bisphosphonates in patients with NETs varies widely across different institutions given an apparent lack of survival benefit associated with this form of bone-directed treatment $[7,8,65]$. Consistent with reports in other malignancies [66], the monthly administration of bisphosphonates did not show any advantage over less intensive treatment schemes in a small group of patients with NET [13]. Denosumab has been shown to be slightly more effective than bisphosphonates in delaying SREs in patients with bone-metastatic cancers [67]. However, no studies have specifically investigated the efficacy of the RANKL inhibitor in NET patients. While several toxicities including the osteonecrosis of the jaw, hypocalcemia and atypical fractures are shared by both bisphosphonates and denosumab, side effects such as acute phase responses and induction of renal insufficiency may occur when administering bisphosphonates. 
Several questions remain unanswered regarding the treatment landscape of NET bone metastases. First, when should therapy with antiresorptive agents be initiated? Second, should all patients with NET bone metastases receive bone-directed therapies? Third, is there a preferred option among bisphosphonates as well as between bisphosphonates and denosumab? Fourth, is the monthly administration of bisphosphonate inherently superior to the 3-month schedule, in a population of patients with indolent malignancies and long survival times? Although in the absence of evidence guiding clinical decisions, a possible flowchart outlining the treatment algorithm to be potentially followed when facing patients with NET bone metastases is depicted in Figure 3.

\section{Conclusions and Future Perspectives}

The skeleton is the third most frequent site of metastasis in patients with NETs, but the molecular mechanisms leading to the development of bone lesions in these malignancies have been poorly investigated so far. In particular, it is presently unclear whether the dismal outcomes observed in patients with bone-colonizing NETs are the result of an inherently aggressive tumor biology, rather than the consequence of the late onset or late diagnosis of a frequently overlooked condition. The recent advent of ${ }^{68} \mathrm{Ga}$-PET/CT has substantially improved our ability to detect NET bone metastases, and future studies will shed light onto the real frequency of skeletal colonization in NET patients, as well as onto the impact of its earlier recognition on survival outcomes.

Innovative bone-directed treatment approaches are needed to both prevent the occurrence of NET bone metastases and inhibit their growth. In recent years, the evidence that bone-targeting agents interfere with several key steps throughout the process of skeletal colonization prompted the initiation of clinical trials investigating the efficacy of adjuvant bisphosphonates or denosumab in patients with osteotropic cancers [19]. While breast cancer experts currently recommend the use of adjuvant bisphosphonates in selected clinical scenarios, the potential utility of this approach in delaying both skeletal and systemic recurrences in patients with NETs is presently unknown. New bone-targeting agents including cathepsin K inhibitors, c-Src antagonists, antisclerostin antibodies, inhibitors of dickkopf-related protein-1 and activin-A blockers are under active clinical scrutiny [19], although studies specifically focusing on the NET population are presently lacking. Bone-targeting radiopharmaceuticals have been extensively tested in patients with bone-devastating cancers, and the calcium-mimetic radium-223 dichloride has been approved for patients with castration-resistant prostate cancer and skeletal metastases [68]. Given that radium-223 is able to form hydroxyapatite complexes in areas of high osteoblast activity, and that NET bone metastases are prevalently osteosclerotic, future research should define the role of this $\alpha$-emitter in patients with bone-colonizing NETs. Key areas to be prioritized by clinical research in the next few years include the prospective evaluation of the impact of PRRT on NET bone lesion growth, the definition of the optimal dosing interval of bisphosphonates in NET bone metastases, the longitudinal assessment of quality of life in patients treated or not with bonetargeting agents, as well as the identification of potential subgroups of patients who may negligibly benefit of bonedirected interventions, thus requiring only observation.

While we envisage that the advent of precision medicine will help us identifying NET patients more likely to develop bone metastases and stratifying them according to their SRE risk, the development of more sophisticated diagnostic and therapeutic modalities will progressively improve our ability to detect, prevent and treat NET bone metastases. In the meantime, clinical wisdom is needed when facing NET patients with skeletal lesions.

\section{Acknowledgment}

The authors thank Dr. Lucrezia Alemanno for editorial assistance.

\section{Disclosure Statement}

The authors declare no affiliation with industries or organizations with a financial interest, direct or indirect, that may affect the conduct or reporting of the work submitted.

\section{Funding Sources}

This work was supported by a grant from the Italian Association for Cancer Research (AIRC, MFAG23583; Mauro Cives) and Apulia Region (Oncogenomic Project, 2015; Franco Silvestris).

\section{Author Contributions}

Dr. Cives and Dr. Panzuto have conceived the manuscript. Dr. Cives, Dr. Pellè, Dr. Rinzivillo, Dr. Prosperi and Dr. Panzuto have participated in manuscript writing. Prof. Tucci and Prof. Silvestris have reviewed the manuscript draft. 


\section{References}

1 Cives M, Strosberg JR. Gastroenteropancreatic neuroendocrine tumors. CA Cancer J Clin. 2018 Nov;68(6):471-87.

2 Dasari A, Shen C, Halperin D, Zhao B, Zhou $\mathrm{S}, \mathrm{Xu} \mathrm{Y}$, et al. Trends in the Incidence, Prevalence, and Survival Outcomes in Patients With Neuroendocrine Tumors in the United States. JAMA Oncol. 2017 Oct;3(10):133542.

3 Klimstra DS, Kloppel G, La Rosa S, Rindi G. Classification of neuroendocrine neoplasms of the digestive system. WHO classification of tumors of the digestive system. Volume 1. 5th ed. Lyon, France: IARC Press; 2019.

4 Riihimäki M, Hemminki A, Sundquist K, Sundquist J, Hemminki K. The epidemiology of metastases in neuroendocrine tumors. Int J Cancer. 2016 Dec;139(12):2679-86.

5 Pape UF, Berndt U, Müller-Nordhorn J, Böhmig M, Roll S, Koch M, et al. Prognostic factors of long-term outcome in gastroenteropancreatic neuroendocrine tumours. Endocr Relat Cancer. 2008 Dec;15(4):1083-97.

6 Nuñez-Valdovinos B, Carmona-Bayonas A, Jimenez-Fonseca P, Capdevila J, CastañoPascual Á, Benavent M, et al. Neuroendocrine Tumor Heterogeneity Adds Uncertainty to the World Health Organization 2010 Classification: Real-World Data from the Spanish Tumor Registry (R-GETNE). Oncologist. 2018 Apr;23(4):422-32.

7 Scharf M, Petry V, Daniel H, Rinke A, Gress TM. Bone Metastases in Patients with Neuroendocrine Neoplasm: Frequency and Clinical, Therapeutic, and Prognostic Relevance. Neuroendocrinology. 2018;106(1):30-7.

8 Kavecansky J, Wei L, Caronia L, Ramirez MT, Bloomston M, Shah MH. Bone metastases in well-to-moderately differentiated neuroendocrine tumors: a single institutional review from the Ohio State University Medical Center. Pancreas. 2015 Mar;44(2):198-203.

9 Van Loon K, Zhang L, Keiser J, Carrasco C, Glass K, Ramirez MT, et al. Bone metastases and skeletal-related events from neuroendocrine tumors. Endocr Connect. 2015 Mar; 4(1):9-17.

10 Panzuto F, Puscedddu S, Faggiano A, Rinzivillo M, Brighi N, Prinzi N, et al.; Itanet (Italian Association for Neuroendocrine Tumours). Prognostic impact of tumour burden in stage IV neuroendocrine neoplasia: A comparison between pancreatic and gastrointestinal localizations. Pancreatology. 2019 Dec; 19(8):1067-73.

11 Cives M, Rizzo F, Simone V, Bisceglia F, Stucci S, Seeber A, et al. Reviewing the Osteotropism in Neuroendocrine Tumors: The Role of Epithelial-Mesenchymal Transition. Neuroendocrinology. 2016;103(3-4):321-34.

12 Strosberg J, Gardner N, Kvols L. Survival and prognostic factor analysis of 146 metastatic neuroendocrine tumors of the mid-gut. Neuroendocrinology. 2009;89(4):471-6.
13 Alexandraki KI, Pizanias M, Uri I, Thomas D, Page T, Kolomodi D, et al. The prognosis and management of neuroendocrine neoplasmsrelated metastatic bone disease: lessons from clinical practice. Endocrine. 2019 Jun;64(3): 690-701.

14 Peri M, Botteri E, Pisa E, De Marinis F, Ungaro A, Spada F, et al. A single-institution retrospective analysis of metachronous and synchronous metastatic bronchial neuroendocrine tumors. J Thorac Dis. 2018 Jul;10(7): 3928-39.

15 Zheng Z, Chen C, Jiang L, Zhou X, Dai X, Song $\mathrm{Y}$, et al. Incidence and risk factors of gastrointestinal neuroendocrine neoplasm metastasis in liver, lung, bone, and brain: A population-based study. Cancer Med. 2019 Dec; 8(17):7288-98.

16 Lombard-Bohas C, Mitry E, O’Toole D, Louvet C, Pillon D, Cadiot G, et al.; FFCD-ANGH-GERCOR. Thirteen-month registration of patients with gastroenteropancreatic endocrine tumours in France. Neuroendocrinology. 2009;89(2):217-22.

17 Robelin P, Hadoux J, Forestier J, Planchard D, Hervieu V, Berdelou A, et al. Characterization, Prognosis, and Treatment of Patients With Metastatic Lung Carcinoid Tumors. J Thorac Oncol. 2019 Jun;14(6):993-1002.

18 Ross EM, Roberts WC. The carcinoid syndrome: comparison of 21 necropsy subjects with carcinoid heart disease to 15 necropsy subjects without carcinoid heart disease. Am J Med. 1985 Sep;79(3):339-54.

19 D'Oronzo S, Coleman R, Brown J, Silvestris F. Metastatic bone disease: pathogenesis and therapeutic options: update on bone metastasis management. J Bone Oncol. 2018;15:0044.

20 Lambert AW, Pattabiraman DR, Weinberg RA. Emerging biological principles of metastasis. Cell. 2017 Feb;168(4):670-91.

21 Fendrich V, Waldmann J, Esni F, Ramaswamy A, Mullendore M, Buchholz M, et al. Snail and Sonic Hedgehog activation in neuroendocrine tumors of the ileum. Endocr Relat Cancer. 2007 Sep;14(3):865-74.

22 Fendrich V, Maschuw K, Waldmann J, Buchholz M, Rehm J, Gress TM, et al. Epithelialmesenchymal transition is a critical step in tumorgenesis of pancreatic neuroendocrine tumors. Cancers (Basel). 2012 Mar;4(1):28194.

23 Galván JA, Astudillo A, Vallina A, Fonseca PJ, Gómez-Izquierdo L, García-Carbonero R, et al. Epithelial-mesenchymal transition markers in the differential diagnosis of gastroenteropancreatic neuroendocrine tumors. Am J Clin Pathol. 2013 Jul;140(1):61-72.

24 Galván JA, Astudillo A, Vallina A, Crespo G, Folgueras MV, González MV. Prognostic and diagnostic value of epithelial to mesenchymal transition markers in pulmonary neuroendocrine tumors. BMC Cancer. 2014 Nov;14(1): 855.
25 Ikezono Y, Koga H, Akiba J, Abe M, Yoshida T, Wada F, et al. Pancreatic Neuroendocrine Tumors and EMT Behavior Are Driven by the CSC Marker DCLK1. Mol Cancer Res. 2017 Jun;15(6):744-52.

26 Kim JY, Lee SH, An S, Kim SJ, Sung YN, Song $\mathrm{KB}$, et al. Carbonic anhydrase 9 expression in well-differentiated pancreatic neuroendocrine neoplasms might be associated with aggressive behavior and poor survival. Virchows Arch. 2018 May;472(5):739-48.

27 Yonemori K, Kurahara H, Maemura K, Mataki Y, Sakoda M, Iino S, et al. Impact of Snail and E-cadherin expression in pancreatic neuroendocrine tumors. Oncol Lett. 2017 Aug; 14(2):1697-702.

28 Cives M, Quaresmini D, Rizzo FM, Felici C, D'Oronzo S, Simone V, et al. Osteotropism of neuroendocrine tumors: role of the CXCL12/ CXCR4 pathway in promoting EMT in vitro. Oncotarget. 2017 Apr;8(14):22534-49.

29 Pellé E, Cives M, Quaresmini D, Lovero D, Felici C, Cafforio P, et al. CXCR4 inhibition by ulocuplumab prevents EMT of pNET cells in vitro. Ann Oncol. 2017;28 Suppl 5:142-57.

30 Arvidsson Y, Bergström A, Arvidsson L, Kristiansson E, Ahlman H, Nilsson O. Hypoxia stimulates CXCR4 signalling in ileal carcinoids. Endocr Relat Cancer. 2010 Jun;17(2): 303-16.

31 Speisky D, Duces A, Bièche I, Rebours V, Hammel P, Sauvanet A, et al.; GTE Group, Groupe d'Etude des Tumeurs Endocrines. Molecular profiling of pancreatic neuroendocrine tumors in sporadic and Von HippelLindau patients. Clin Cancer Res. 2012 May; 18(10):2838-49.

32 Xu SS, Xu HX, Wang WQ, Li S, Li H, Li TJ, et al. Tumor-infiltrating platelets predict postoperative recurrence and survival in resectable pancreatic neuroendocrine tumor. World J Gastroenterol. 2019 Nov;25(41): 6248-57.

33 Peinado H, Zhang H, Matei IR, Costa-Silva B, Hoshino A, Rodrigues G, et al. Pre-metastatic niches: organ-specific homes for metastases. Nat Rev Cancer. 2017 May;17(5):302-17.

34 Tucci M, Mannavola F, Passarelli A, Stucci LS, Cives M, Silvestris F. Exosomes in melanoma: a role in tumor progression, metastasis and impaired immune system activity. Oncotarget. 2018 Apr;9(29):20826-37.

35 Błogowski W, Zuba-Surma E, Sałata D, Budkowska M, Dołęgowska B, Starzyńska T. Peripheral trafficking of bone-marrow-derived stem cells in patients with different types of gastric neoplasms. OncoImmunology. 2015 Oct;5(4):e1099798.

36 Khan MS, Tsigani T, Rashid M, Rabouhans JS, Yu D, Luong TV, et al. Circulating tumor cells and EpCAM expression in neuroendocrine tumors. Clin Cancer Res. 2011 Jan; 17(2):337-45. 
37 Khan MS, Kirkwood A, Tsigani T, GarciaHernandez J, Hartley JA, Caplin ME, et al. Circulating tumor cells as prognostic markers in neuroendocrine tumors. J Clin Oncol. 2013 Jan;31(3):365-72.

38 Hsieh JC, Chen GY, Jhou DD, Chou WC, Yeh $\mathrm{CN}$, Hwang TL, et al. The Prognostic Value of Circulating Tumor Cells in Asian Neuroendocrine Tumors. Sci Rep. 2019 Dec;9(1): 19917.

39 Rizzo FM, Vesely C, Childs A, Marafioti T, Khan MS, Mandair D, et al. Circulating tumour cells and their association with bone metastases in patients with neuroendocrine tumours. Br J Cancer. 2019 Feb;120(3):294300.

40 Massagué J, Obenauf AC. Metastatic colonization by circulating tumour cells. Nature. 2016 Jan;529(7586):298-306.

41 Weilbaecher KN, Guise TA, McCauley LK. Cancer to bone: a fatal attraction. Nat Rev Cancer. 2011 Jun;11(6):411-25.

42 Milone F, Pivonello C, Cariati F, Sarnataro M, Ramundo V, Marotta V, et al. Assessment and clinical implications of RANK/RANKL/OPG pathway as markers of bone tumor progression in patients with NET harboring bone metastases. Biomarkers. 2013 Mar;18(2):1215.

43 Merola E, Pavel ME, Panzuto F, Capurso G, Cicchese N, Rinke A, et al. Functional Imaging in the Follow-Up of Enteropancreatic Neuroendocrine Tumors: Clinical Usefulness and Indications. J Clin Endocrinol Metab. 2017 May;102(5):1486-94.

44 Sadowski SM, Neychev V, Millo C, Shih J, Nilubol N, Herscovitch P, et al. Prospective study of $68 \mathrm{Ga}$-DOTATATE positron emission tomography/computed tomography for detecting gastro-entero-pancreatic neuroendocrine tumors and unknown primary sites. J Clin Oncol. 2016 Feb;34(6):588-96.

45 Lim KHJ, Raja H, D'Arienzo P, Barriuso J, McNamara MG, Hubner RA, et al. Identification of areas for improvement in the management of bone metastases in patients with neuroendocrine neoplasms. Neuroendocrinology. 2020;110:688-96.

46 Shi W, Johnston CF, Buchanan KD, Ferguson WR, Laird JD, Crothers JG, et al. Localization of neuroendocrine tumours with [111In] DTPA-octreotide scintigraphy (Octreoscan): a comparative study with CT and MR imaging. QJM. 1998 Apr;91(4):295-301.

47 Gauthé M, Testart Dardel N, Ruiz Santiago F, Ohnona J, Nataf V, Montravers F, et al. Vertebral metastases from neuroendocrine tumours: how to avoid false positives on $68 \mathrm{Ga}$ DOTA-TOC PET using CT pattern analysis? Eur Radiol. 2018 Sep;28(9):3943-52.
48 Albanus DR, Apitzsch J, Erdem Z, Erdem O, Verburg FA, Behrendt FF, et al. Clinical value of ${ }^{68} \mathrm{Ga}$-DOTATATE-PET/CT compared to stand-alone contrast enhanced CT for the detection of extra-hepatic metastases in patients with neuroendocrine tumours (NET). Eur J Radiol. 2015 Oct;84(10):1866-72.

49 Ambrosini V, Nanni C, Zompatori M, Campana D, Tomassetti P, Castellucci P, et al. (68) Ga-DOTA-NOC PET/CT in comparison with CT for the detection of bone metastasis in patients with neuroendocrine tumours. Eur J Nucl Med Mol Imaging. 2010 Apr;37(4): $722-7$.

50 Crown A, Rocha FG, Raghu P, Lin B, Funk G, Alseidi A, et al. Impact of initial imaging with gallium-68 dotatate PET/CT on diagnosis and management of patients with neuroendocrine tumors. J Surg Oncol. 2020 Mar;121(3):4805

51 Skoura E, Michopoulou S, Mohmaduvesh M, Panagiotidis E, Al Harbi M, Toumpanakis C, et al. The impact of 68Ga-DOTATATE PET/ $\mathrm{CT}$ imaging on management of patients with neuroendocrine tumors: experience from a national referral center in the United Kingdom. J Nucl Med. 2016 Jan;57(1):34-40.

52 Jiménez-Fonseca P, Krug S, Tamagno G, Fierro Maya F, Monléon Getino A, Rodriguez Casado CI, et al. Identifying Prognostic Factors for Well-Differentiated Metastatic Pancreatic Neuroendocrine Tumours: A Retrospective International Multicentre Cohort Study. Neuroendocrinology. 2018;107(4): 315-23.

53 Panzuto F, Merola E, Rinzivillo M, Partelli S, Campana D, Iannicelli E, et al. Advanced digestive neuroendocrine tumors: metastatic pattern is an independent factor affecting clinical outcome. Pancreas. 2014 Mar;43(2): 212-8.

54 Carmona-Bayonas A, Jiménez-Fonseca P, Lamarca Á, Barriuso J, Castaño Á, Benavent M, et al. Prediction of Progression-Free Survival in Patients With Advanced, Well-Differentiated, Neuroendocrine Tumors Being Treated With a Somatostatin Analog: the GETNETRASGU Study. J Clin Oncol. 2019 Oct; 37(28):2571-80.

55 Benslama N, Bollard J, Vercherat C, Massoma $\mathrm{P}$, Roche C, Hervieu V, et al. Prediction of response to everolimus in neuroendocrine tumors: evaluation of clinical, biological and histological factors. Invest New Drugs. 2016 Oct;34(5):654-62.

56 Strosberg J, El-Haddad G, Wolin E, Hendifar A, Yao J, Chasen B, et al; NETTER-1 Trial Investigators. Phase 3 trial of 177Lu-Dotatate for midgut neuroendocrine tumors. N Engl J Med. 2017 Jan;376(2):125-35.

57 Sabet A, Khalaf F, Haslerud T, Al-Zreiqat A, Sabet A, Simon B, et al. Bone metastases in GEP-NET: response and long-term outcome after PRRT from a follow-up analysis. Am J Nucl Med Mol Imaging. 2013 Sep;3(5):43745.
58 Ezziddin S, Sabet A, Heinemann F, YongHing CJ, Ahmadzadehfar H, Guhlke S, et al. Response and long-term control of bone metastases after peptide receptor radionuclide therapy with (177)Lu-octreotate. J Nucl Med. 2011 Aug;52(8):1197-203.

59 Cives M, Strosberg J. Radionuclide Therapy for Neuroendocrine Tumors. Curr Oncol Rep. 2017 Feb;19(2):9.

60 van Vliet EI, Hermans JJ, de Ridder MA Teunissen JJ, Kam BL, de Krijger RR, et al. Tumor response assessment to treatment with [177Lu-DOTA0,Tyr3]octreotate in patients with gastroenteropancreatic and bronchial neuroendocrine tumors: differential response of bone versus soft-tissue lesions. J Nucl Med. 2012 Sep;53(9):1359-66.

61 Guan M, He I, Luu M, David J, Gong J, Placencio-Hickok VR, et al. Palliative Radiation Therapy for Bone Metastases in Neuroendocrine Neoplasms. Adv Radiat Oncol. 2019 Apr;4(3):513-9.

62 Yalchin $\mathrm{M}$, Tharmalingam $\mathrm{H}$, Owen C, Walker M, Caplin M, Stewart G, et al. The role of external beam radiotherapy in the management of advanced neuroendocrine tumors. Presented at the 12th Annual ENETS Conference, Barcelona, Spain. Neuroendocrinology. 2015; 102:136.

63 Lutz S, Balboni T, Jones J, Lo S, Petit J, Rich $\mathrm{SE}$, et al. Palliative radiation therapy for bone metastases: Update of an ASTRO EvidenceBased Guideline. Pract Radiat Oncol. 2017 Jan - Feb;7(1):4-12.

64 Kos-Kudła B, O’Toole D, Falconi M, Gross D, Klöppel G, Sundin A, et al.; Palma de Mallorca Consensus Conference Participants. ENETS consensus guidelines for the management of bone and lung metastases from neuroendocrine tumors. Neuroendocrinology. 2010;91(4):341-50.

65 Altieri B, Di Dato C, Martini C, Sciammarella C, Di Sarno A, Colao A, et al.; NIKE Group. Bone Metastases in Neuroendocrine Neoplasms: From Pathogenesis to Clinical Management. Cancers (Basel). 2019 Sep; 11(9):E1332.

66 Himelstein AL, Foster JC, Khatcheressian JL, Roberts JD, Seisler DK, Novotny PJ, et al. Effect of Longer-Interval vs Standard Dosing of Zoledronic Acid on Skeletal Events in Patients With Bone Metastases: A Randomized Clinical Trial. JAMA. 2017 Jan;317(1):48-58.

67 Lipton A, Fizazi K, Stopeck AT, Henry DH, Brown JE, Yardley DA, et al. Superiority of denosumab to zoledronic acid for prevention of skeletal-related events: a combined analysis of 3 pivotal, randomised, phase 3 trials. Eur J Cancer. 2012 Nov;48(16):3082-92.

68 Parker C, Nilsson S, Heinrich D, Helle SI, O'Sullivan JM, Fosså SD, et al; ALSYMPCA Investigators. Alpha emitter radium-223 and survival in metastatic prostate cancer. $N$ Engl J Med. 2013 Jul;369(3):213-23. 